\title{
Fisheye lens design for solar-powered mobile ultrasound devices
}

\author{
Seonho Ryu ${ }^{\mathrm{a}}$, Jaemyung Ryu ${ }^{\mathrm{a}, *}$ and Hojong Choi ${ }^{\mathrm{b}, *}$ \\ ${ }^{a}$ Department of Optical System Engineering, Kumoh National Institute of Technology, Gumi, Korea \\ ${ }^{\mathrm{b}}$ Department of Electronic Engineering, Gachon University, Seongnam, Korea
}

\begin{abstract}
.
BACKGROUND: Compared to benchtop ultrasound machines, mobile ultrasound machines require portable batteries when acquiring information regarding human tissues during outdoor activities.

OBJECTIVE: A novel fisheye lens type was designed to address the charging issue where it is difficult to constantly track the sun. This method does not require the use of a mechanical motor that constantly tracks the sun to charge the portable batteries. METHODS: To obtain an optical solar power system, the numerical aperture (NA) and field angle must be increased. Therefore, we use the fisheye lens with the largest field angle.

RESULTS: The NA of the designed fisheye lens system reaches 0.75 , allowing light collection of approximately $\pm 48^{\circ}$. Additionally, the efficiency ratio of the central and surrounding areas also satisfies more than $80 \%$ at a field angle of $85^{\circ}$ and more than $70 \%$ at field angles of $85^{\circ}$ to $90^{\circ}$, respectively.

CONCLUSIONS: We designed a novel fisheye lens for solar-powered mobile ultrasound machines used outdoors.
\end{abstract}

Keywords: Mobile ultrasound machines, fisheye lens, solar power

\section{Introduction}

Ultrasound machines are typically used to check the conditions of patients or cracks in steel for non-destructive testing or applying acoustic power for clinical or surgical purposes [1,2]. Compared with computed X-ray, tomography, positron emission tomography, and magnetic resonant imaging, ultrasound machines are relatively inexpensive imaging modalities [2-5]. Currently, mobile ultrasound machines are widely used for emergency rooms in hospitals or outdoor activities such as sports owing to advanced semiconductor technologies [6-9]. Mobile ultrasound machines with battery modules can be utilized without any power cords and can remotely send the imaging data using communication channels [10-12]. The received imaging data can be checked by clinical specialist for immediate diagnosis. This could be very useful for emergency cases or prevention of side effects [13]. Compared with benchtop ultrasound machines used in clinics or hospitals, mobile ultrasound machines are suitable for outdoor activity owing to portable batteries [14,15]. Because of their limited battery lives, mobile ultrasound machines should be charged in advance for emergency cases during application in high altitude regions such as

\footnotetext{
${ }^{*}$ Corresponding authors: Jaemyung Ryu, Department of Optical System Engineering, Kumoh National Institute of Technology, 35027 Gumi-Daero, Gumi 39253, Korea. E-mail: jmryu@kumoh.ac.kr. Hojong Choi, Department of Electronic Engineering, Gachon University, 13 420, Seongnam-daero, Sujeong-gu, Seongnam-si, Gyeonggi-do, Korea. E-mail: hojongch@gachon.ac.kr.
} 


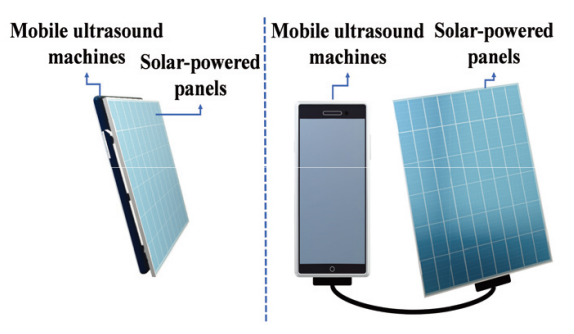

(a)

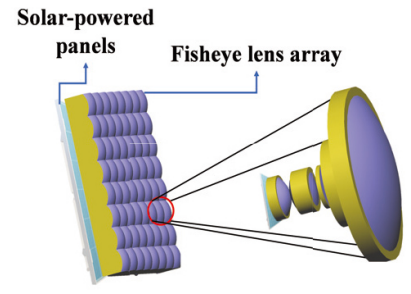

(b)

Fig. 1. Conceptual block diagram of (a) mobile ultrasound machines attached to or separated from the solar-powered panels and (b) designed fisheye lens optical system for mobile ultrasound machines.

mountains [16]. Therefore, mobile ultrasound companies provide solar-power panels attached behind the machines or solar-panel devices separated from the ultrasound machines [17,18].

Solar-powered panels utilize mechanical motors requiring additional power for the reflectors [19]. The mechanical motors generate noise that cannot be removed during image processing for patient images [20,21]. Therefore, the unwanted noise signals directly affect the imaging quality of the mobile ultrasound machines [22-25]. Additionally, mobile ultrasound machines have lower image quality than the benchtop ones because of the reduced number of ultrasound probe channels partially caused by the portable batteries [26-29]. This is because ultrasound companies should consider the available transducer channels because of excessive heat [30-32]. To replace the mechanical motors that track the sun to charge the batteries, a fisheye lens was designed to receive sunlight efficiently. A reflector-tracking system was used to track the sun in the solar power generation [23]. However, a mechanical motor is required to rotate the reflector thus electricity is consumed to drive the motor. To constantly receive sunlight without the reflector, we designed a fisheye optical lens which requires a large NA. Figure 1(a) shows the block diagram of two typical types of mobile ultrasound machines attached to or separated from solar-powered panels. Figure 1(b) shows the designed fisheye lens. As shown in Fig. 1(b), the fisheye lens modules are designed to receive sunlight without the reflector. In the optical system, the converter is designed with a spherical lens, and the aspherical surface for relay optics can be applied to an inexpensive material for automobile head lamps.

\section{Methods}

The optical system used for the mobile ultrasound machine proposed in this study should have a high NA. To generate power for an extended period of time, light must be received regardless of the altitude of the sun; therefore, the field angle of the optical system must be wide [33-36]. A fisheye lens with a very wide field angle and high NA should be designed to achieve this [37]. To design an optical system that satisfies the given specifications, a converter system capable of converting the field angle is designed. Here, optical systems with a typical field angle which are coupled behind the stop are referred to as relay optics. Therefore, the converter system must be positioned in front of the stop, and the distance between the two optical systems must be adjusted so that the exit pupil of the converter and entrance pupil of the relay optics to be combined are matched. Particularly, the converter in front of the stop converts light with a half field angle of $90^{\circ}$ into a small field incident angle, and the relay optics behind the stop are designed and combined to have a high NA. When combining two optics, the angle of the chief ray passing through the converter and entering the stop should match the field angle of the relay optics [38]. Figure 2 shows 


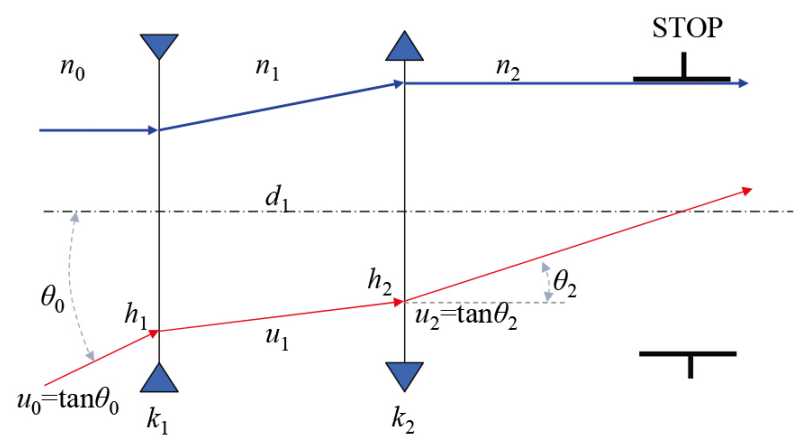

Fig. 2. Paraxial layout for converter system.

the paraxial layout of a converter in which lenses with negative and positive refractive power values are combined [39]. Therefore, the refractive power of the optical converter should be zero, and the angle of the chief ray decreases from $\theta$ to $\theta_{2}$.

In Fig. $2, n_{0}, n_{1}$, and $n_{2}$ represent the refractive indices at the object plane, between the two lenses, and at the stop, respectively. Considering that the optical system is used in air, these values are all equal to 1 . The distance between the two lenses is $d_{1}$, and the refractive power values of the two lenses are $k_{1}$ and $k_{2}$, respectively. Therefore, the refractive power of this system is given by Eq. (1). Simultaneously, the chief ray is incident on $\theta_{0}$, and the height of the first surface is $h_{1}$ that is, $u_{0}=\tan \theta_{2}$. When the chief ray passes through the first lens, the refraction angle $u_{1}$ is calculated using the refraction equation shown in Eq. (2). After refraction from the first lens, the height $h_{2}$ is expressed as Eq. (3) if the chief ray travels by $d_{1}$. Further, the chief ray is refracted by the second lens, resulting in $u_{2}$ as shown in Eq. (4). Here, the angle of the chief ray passing through the optical system is $\theta_{2}$ that is, $u_{2}=\tan \theta_{2}$. Equation (4) is the conversion equation of the light rays passing through the converter lens by substituting Eqs (2) and (3). Because the angular magnification can be defined as the ratio of the incident and exit angles of the chief ray, it can be expressed as Eq. (5), and can be obtained by substituting Eqs (1) and (4) into Eq. (5). Substituting Eq. (5) into Eq. (1) again, the refractive power of each lens constituting the converter lens can be calculated as in Eq. (6). Therefore, as shown in Eq. (6), the refractive power for each lens of the converter is determined by the angular magnification $m$ and distance $d_{1}$ between the lenses [40,41].

$$
\begin{aligned}
& K=k_{1}+k_{2}-\frac{d_{1}}{n_{1}} k_{1} k_{2}=0=\frac{1}{E F L} \\
& h_{2}=h_{1}+d_{1} u_{1} \\
& n_{1} u_{1}=n_{0} u_{0}-h_{1} k_{1} \\
& n_{2} u_{2}=n_{1} u_{1}-h_{2} k_{2}=n_{0} u_{0}\left(1-k_{2} \frac{d_{1}}{n_{1}}\right)-h_{1} K \\
& m=\frac{n_{2} u_{2}}{n_{0} u_{0}}=\frac{n_{2} \tan \theta_{2}}{n_{0} \tan \theta_{0}}=1-k_{2} \frac{d_{1}}{n_{1}}=-\frac{k_{2}}{k_{1}}(\because K=0) \\
& \therefore k_{1}=\frac{m-1}{d \cdot m} \quad k_{2}=-\frac{m-1}{d}
\end{aligned}
$$

The refractive power of the two lenses of the optical system shown in Fig. 2 can be calculated by Eq. (6). Therefore, the angular magnification $m$ is determined by the angle to be converted in the optical system, and the refractive power of the two lenses can be obtained if the distance $d_{1}$ between the two 


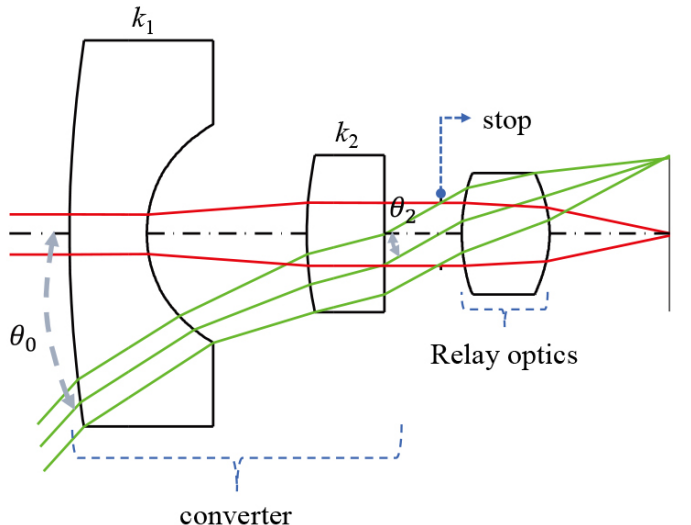

(a)

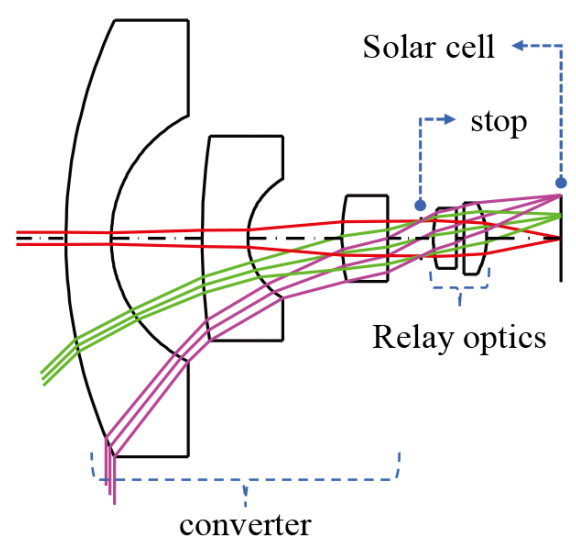

(b)

Fig. 3. (a) Optical layout for our designed optics and (b) paraxial system with added lenses in Fig. 3(a).

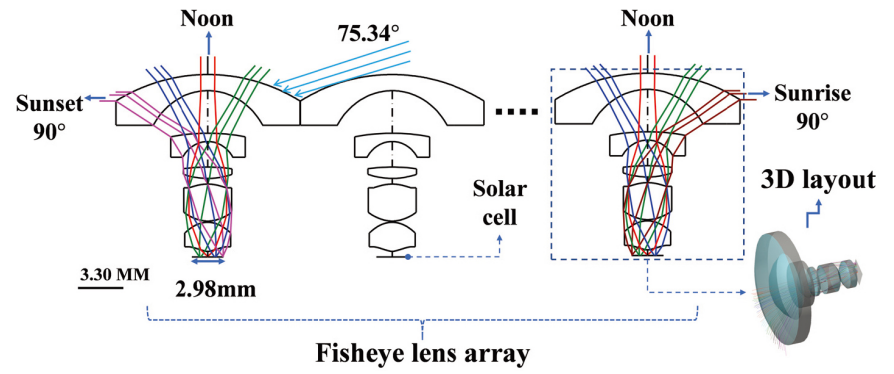

Fig. 4. Optical layout for our finally designed fish-eye system.

lenses is properly determined. Substituting $m$ with 0.6 and $d_{1}=2.0 \mathrm{~mm}$ into Eq. (6), $k_{1}$ and $k_{2}$ become $0.333 \mathrm{~mm}^{-1}$ and $k_{1}=+0.200 \mathrm{~mm}^{-1}$, respectively. The converter and singlet calculated using this method are combined, and the result is shown in Fig. 3(a). Here, the singlet was designed by the method mentioned in [42-44]. In the optical system shown in Fig. 3(a), a lens was added because the field angle does not satisfy $90^{\circ}$. Because chromatic aberration may occur in the singlet corresponding to the relay optics, the singlet was divided and converted into an air-spaced doublet. This way, the equivalent lens method was used to divide the singlet into a doublet [45]. The layout of the optical system in which this method is applied is shown in Fig. 3(b). The paraxial design (Fig. 3(b)) does not satisfy sufficient optical performance. To increase the power generation, the entrance pupil diameter (EPD) of the optical system as shown in Fig. 3(b) must be increased. A large EPD indicates a large NA. Therefore, it is necessary to proceed with the optical design so that the NA is increased and the spot size on the image surface is minimized.

\section{Results and discussion}

Figure 4 shows the layout of the optical system that satisfies the optical performance. For this optical system, the effective field length (EFL) is $0.885 \mathrm{~mm}$, NA is 0.750 , and the overall length of the optical system is approximately $16.1 \mathrm{~mm}$. The light is collected in a solar cell with the optical system as shown 

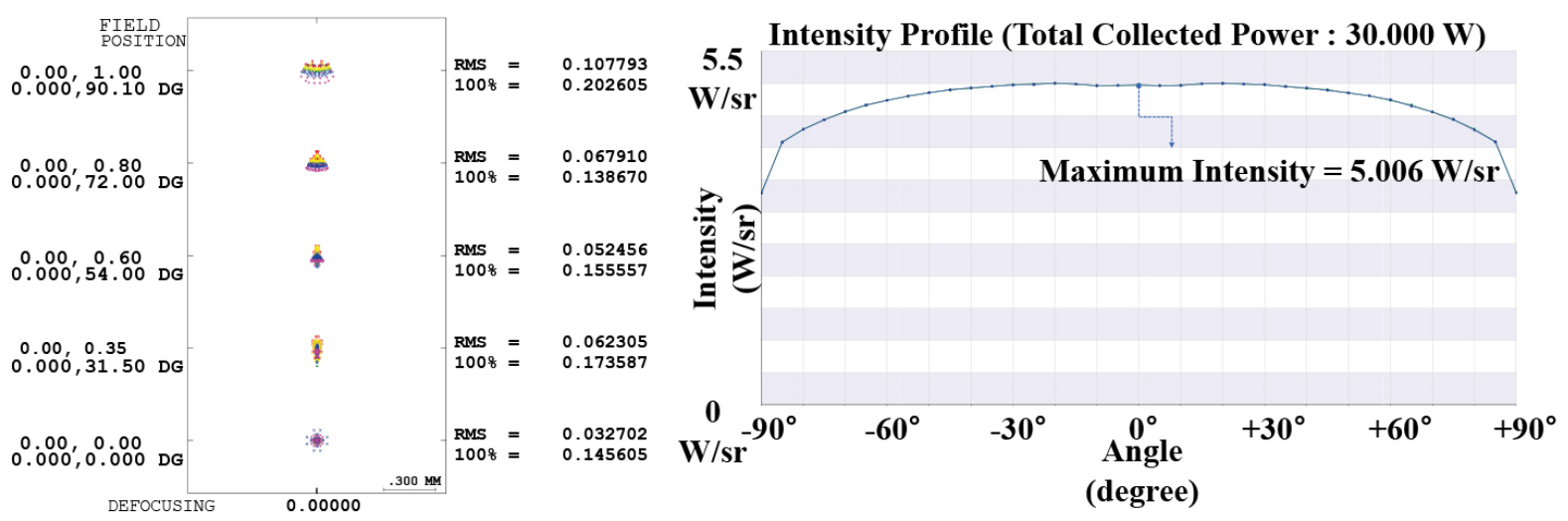

Fig. 5. (a) Spot diagram of our finally designed fish-eye system. (b) Power graph results in the optical system for each field angle. The DG and RMS represent the degree and root mean square, respectively.

in Fig. 4. Therefore, several such modules can be installed if the amount of power generated by one solar cell is not sufficient.

Figure 5(a) shows the spot diagram in the optical system where the optical design is completed as shown in Fig. 4. The spot diagram refers to the distribution of the ray bundle passing through the optical system for each field angle. The minimum size of the solar panel cell is determined from the height of the chief ray and spot size. The height of the chief ray is $1.49 \mathrm{~mm}$ as shown in Fig. 4, and it can be observed that the spot size is approximately 0.14 to $0.21 \mathrm{~mm}$ as shown in Fig. 5(b). Therefore, the cell size is determined by the sum of the height of the chief ray and spot size. Because the field angle in which light is incident is $\pm 90^{\circ}$, the size of the solar cell must be $3.42 \times 3.42 \mathrm{~mm}$ or more. Power generation is possible for our designed optical system regardless of the incident angle of the sunlight.

To compare the power generation produced by this optical system over time, we presented the result shown in Fig. 5(b) which is a power graph based on the field angle. The ratio of the power generation at the center and periphery is more than $80 \%$ at approximately $80^{\circ}$ and more than $70 \%$ at $90^{\circ}$. This indicates that the ratio of the minimum generation in the morning and afternoon compared to noon is more than $70 \%$. There is a difference in the amount of light between noon sunrise or sunset; therefore, the actual power generation over time is expected to be lower than the obtained results. However, because the FOV is $\pm 90^{\circ}$, power generation is possible without moving the panel or optical system in the direction of the light. If the array is configured as shown in Fig. 1, light with a field angle of $\pm 90^{\circ}$ may not be incident on the solar cell. When configured as an array, the field angle incident on the solar cell is about $\pm 74.341^{\circ}$ as shown in Fig. 4. If it is located around the array, light of $\pm 90^{\circ}$ is incident. Thus, the optical design was performed based on the field angle of $\pm 90^{\circ}$.

A novel fisheye lens type was designed to overcome the charging problem of constantly tracking the sun. With these optics, it is not necessary to use a mechanical motorized power generation system to track the altitude of the existing sun.

\section{Conclusion}

Conventional systems used for solar power generation use parabolic mirrors. The parabolic mirror has a very small field angle and can only be used at a specific time. To overcome this, the parabolic mirror must be applied alongside a mechanical motor to track the sun. However, a system with a mechanical 
motor uses some of the energy generated to drive the mechanical motor. Therefore, a fisheye lens that can receive light from all field angles was used to solve this problem. To increase the amount of power generation, the entrance pupil of the fisheye lens must be increased. The optical system must have a high NA to achieve this. An optical system that satisfies these requirements was designed by separating the converter to change the field angle and relay optics to collect light at a spot.

The converter located in front of the aperture is designed so that the incident angle on the aperture is similar to that of the relay optics. However, if a converter is simply used, the field angle does not satisfy $90^{\circ}$, thus a lens is added. Additionally an air-spaced doublet was configured to correct the chromatic aberration of relay optics and increase the NA. For comparison, the amount of power produced by the designed optical system over time was estimated. From the results, the relative illumination of the optical system was $80 \%$ or more up to a field angle of $80^{\circ}$. Therefore, the power generation ratio will be $80 \%$ when the field angle is $0^{\circ}$ and $80^{\circ}$. Therefore, solar power generation is possible at any time, and it is expected that mobile ultrasonic devices can be more effective using our designed fish-eye system.

In cloudy weather, electricity may not be sufficient. However, this problem occurs not only in the system, but also in all solar power systems. In the system of this study, the solar cell and the optical system are integrated. Therefore, it is expected that the amount of power generation can be increased by combining them in an array form.

\section{Acknowledgments}

This research was supported by the Basic Science Research Program through the National Research Foundation of Korea (NRF) funded by the Ministry of Education (NRF-2020R1I1A3052712), and the National Research Foundation of Korea (NRF) grant funded by the Korean government (MSIT) (No. 2020R1A2C4001606).

\section{Conflict of interest}

None to report.

\section{References}

[1] Kim J, You K, Choe S, Choi H. Wireless ultrasound surgical system with enhanced power and amplitude performances. Sensors. 20(15) (2020), 4165.

[2] Kim J, You K, Choi H. Post-voltage-boost circuit-supported single-ended class-b amplifier for piezoelectric transducer applications. Sensors. 20(18) (2020), 5412.

[3] Ullah M, Park C, Pratiwi E, Kim C, Choi H, Yeom J. A new positron-gamma discriminating phoswich detector based on wavelength discrimination (WLD). Nucl. Instrum. Methods Phys. Res., Sect. A. 946 (2019), 162631.

[4] Ullah M, Pratiwi E, Park J, Lee K, Choi H, Yeom J. Wavelength discrimination (WLD) TOF-PET detector with DOI information. Phys. Med. Biol. 65(5) (2019), 055003.

[5] Kim J, Kim K, Choe S, Choi H. Development of an accurate resonant frequency controlled wire ultrasound surgical instrument. Sensors. 20(11) (2020), 3059.

[6] Zawawi R, Abbasi W, Kim S, Choi H, Kim J. Wide-supply-voltage-range CMOS bandgap reference for in vivo wireless power telemetry. Energies. 13(11) (2020), 2986.

[7] Kim K, Choi H. A new approach to power efficiency improvement of ultrasonic transmitters via a dynamic bias technique. Sensors. 21(8) (2021), 2795.

[8] Kim K, Choi H. High-efficiency high-voltage class F amplifier for high-frequency wireless ultrasound systems. PLOS ONE. 16(3) (2021), e0249034. 
[9] Choi H, Choe S. Acoustic stimulation by shunt-diode pre-linearizer using very high frequency piezoelectric transducer for cancer therapeutics. Sensors. 19(2) (2019), 357.

[10] Baston C, Moore C, Dean A, Panebianco N. Pocket Guide to POCUS: Point-of-care Tips for Point-of-care Ultrasound. New York, NJ, USA: McGraw Hill Education, Incorporated, 2019.

[11] Daniels J, Hoppmann R. Practical Point-of-care Medical Ultrasound. New York, NJ, USA: Springer, 2016.

[12] You K, Choi H. Inter-stage output voltage amplitude improvement circuit integrated with class-b transmit voltage amplifier for mobile ultrasound machines. Sensors. 20(21) (2020), 6244.

[13] Shin S, Yoo W, Choi H. Development of public key cryptographic algorithm using matrix pattern for tele-ultrasound applications. Mathematics. 7(8) (2019), 752.

[14] Moore C, Copel J. Point-of-care ultrasonography. N. Engl. J. Med. 364(8) (2011), 749-757.

[15] You K, Kim S, Choi H. A class-j power amplifier implementation for ultrasound device applications. Sensors. 20(8) (2020), 2273.

[16] Wagner M, Garcia K, Martin D. Point-of-care ultrasound in aerospace medicine: Known and potential applications. Aviat Space Environ Med. 85(7) (2014), 730-739.

[17] Karlen W. Mobile point-of-care monitors and diagnostic device design. Boca Raton, FL, USA: CRC Press, 2014.

[18] Nolting L, Baker D, Hardy Z, Kushinka M, Brown H. Solar-powered point-of-care sonography: Our himalayan experience. J. Ultrasound Med. 38(9) (2019), 2477-2484.

[19] Fagenholz P, Murray A, Noble V, Baggish A, Harris N. Ultrasound for high altitude research. Ultrasound Med. Biol. 38(1) (2012), 1-12.

[20] Szabo T. Diagnostic Ultrasound Imaging: Inside Out. London, UK: Elsevier Academic Press, 2013.

[21] Choi H, Yeom J, Ryu J. Development of a multiwavelength visible-range-supported opto-ultrasound instrument using a light-emitting diode and ultrasound transducer. Sensors. 18(10) (2018), 3324.

[22] Choi H. Development of negative-group-delay circuit for high-frequency ultrasonic transducer applications. Sens. Actuators, A. 299 (2019), 111616.

[23] Vignola F, Michalsky J, Stoffel T. Solar and Infrared Radiation Measurements. Hoboken, NJ, USA: CRC press, 2016.

[24] Choi H. Prelinearized class-b power amplifier for piezoelectric transducers and portable ultrasound systems. Sensors. 19(2) (2019), 287.

[25] Ullah M, Park Y, Kim G, Kim C, Park C, Choi H, Yeom J. Simultaneous acquisition of ultrasound and gamma signals with a single-channel readout. Sensors. 21(4) (2021), 1048.

[26] Choi H. Class-c linearized amplifier for portable ultrasound instruments. Sensors. 19(4) (2019), 898.

[27] Istepanian R, Laxminarayan S, Pattichis C. M-health: Emerging mobile health systems. Berlin, Germany: Springer Science \& Business Media, 2007.

[28] Choe S, Choi H. Suppression technique of hela cell proliferation using ultrasonic power amplifiers integrated with a series-diode linearizer. Sensors. 18(12) (2018), 4248.

[29] Choi H, Choe S. Therapeutic effect enhancement by dual-bias high-voltage circuit of transmit amplifier for immersion ultrasound transducer applications. Sensors. 18(12) (2018), 4210.

[30] Choi H. Stacked transistor bias circuit of class-b amplifier for portable ultrasound systems. Sensors. 19(23) (2019), 5252.

[31] You K, Choi H. Wide bandwidth class-s power amplifiers for ultrasonic devices. Sensors. 20(1) (2020), 290.

[32] Choi H. Development of a class-c power amplifier with diode expander architecture for point-of-care ultrasound systems. Micromachines. 10(10) (2019), 697.

[33] Kidger M. Fundamental optical design. 2001: SPIE Bellingham.

[34] Choi G, Jo J, Ryu J. A novel focal length measurement method for center-obstructed omni-directional reflective optical systems. Appl. Sci. 9(11) (2019), 2350.

[35] Seo S, Ryu J, Choi H. Focus-adjustable head mounted display with off-axis system. Applied Sciences. 10(21) (2020), 7931.

[36] Choi H, Ryu J, Yeom J. Development of a double-gauss lens based setup for optoacoustic applications. Sensors. 17(3) (2017), 496.

[37] Choi H, Ryu J, Kim J. A novel fisheye-lens-based photoacoustic system. Sensors. 16(12) (2016), 2185.

[38] Choi Y, Rim C. Design of a telecentric lens with a smartphone camera to utilize machine vision. Korean Journal of Optics and Photonics. 29(4) (2018), 149-158.

[39] Choi H, Choe S, Ryu J. Optical design of a novel collimator system with a variable virtual-object distance for an inspection instrument of mobile phone camera optics. Applied Sciences. 11(8) (2021), 3350.

[40] Choi H, Choe S, Ryu J. A macro lens-based optical system design for phototherapeutic instrumentation. Sensors. 19(24) (2019), 5427.

[41] Choi H, Ryu J, Choe S. A novel therapeutic instrument using an ultrasound-light-emitting diode with an adjustable telephoto lens for suppression of tumor cell proliferation. Measurement. 147 (2019), 106865.

[42] Chee S, Ryu J, Choi H. New optical design method of floating type collimator for microscopic camera inspection. Applied Sciences. 11(13) (2021), 6203 . 
[43] Kim K, Choe S, Ryu J, Choi H. Computation of analytical zoom locus using padé approximation. Mathematics. 8(4) (2020), 581.

[44] Choi H, Ryu J. Design of wide angle and large aperture optical system with inner focus for compact system camera applications. Appl. Sci. 10(1) (2019), 179.

[45] Lee J, Kim K. Generalization of equivalent lens conversion and third order aberration formulae of the generalized equivalent lens system. Korean J. Opt. Photon. (Hankook Kwanghak Hoeji). 7 (1996), 305-313. 